combination therapy with nicotine lozenges with the goal of smoking cessation.

Conclusion. Patients were open to a brief informal intervention targeting smoking behaviours and readily accepted trying nicotine lozenges and prescription during their inpatient stay. The regular use of nicotine replacement therapy by some patients encouraged other patients to try and accept therapy. In addition to the habitual tobacco sharing among patients, nicotine lozenges were also shared especially with newly admitted patients. The evaluation of the impact of this intervention will require a much longer period of time of implementation.

\section{"The path is smooth that leadeth on to danger": caffeine and psychosis}

Isabel Ganhao*, Goncalo Marinho, Afonso Paixa and Miguel Trigo

Centro Hospitalar Psiquiatrico de Lisboa

${ }^{*}$ Corresponding author.

doi: 10.1192/bjo.2021.505

Aims. To review literature on the importance of caffeine intake with regard to psychosis. The need for intervention with regard to caffeine intake hinges on effectively recognizing potential risks. Background. Caffeine is the most widely consumed psychoactive substance worldwide and as such is generally considered acceptable but as a competitive adenosine antagonist, it affects dopamine transmission. Patients with serious mental illness are known to have higher caffeine intakes than the general population. The hierarchy of needs for this patient population is complex, frequently leaving the intake of caffeine under the radar of clinical priorities.

Method. PubMed and Google Scholar search for caffeine/coffee and psychosis/schizophrenia

Result. Of the 43 articles that were considered relevant for clinical practice, caffeine consumption was associated with 1) appearance of psychotic symptoms and episodes (caffeine-induced psychosis) and chronic psychosis in high intake 2) exacerbation of psychosis in schizophrenic patients even in lower intakes, 3) treatment resistance possibly due to interference with antipsychotics (ex. clozapine), 4) abuse and addiction, 5) comorbidity with tobacco smoking and other addictions. Caffeine in low doses was associated with ameliorating cognitive and extrapyramidal side-effects of medication and as a potential treatment strategy for treatment-resistant schizophrenia.

Conclusion. Caffeine consumption may have a greater impact on psychotic symptoms and episodes than is recognized with negative effects outweighing any potential benefits. Greater awareness of the necessity to quantify caffeine intake and implementation of interventions to curb intake may contribute to better quality of care of serious mental illness. Further research is warranted.

\section{Monitoring lithium therapy in a CMHRS during the COVID-19 pandemic}

Katherine Gardner* and Charles Shuttleworth

Surrey and Borders Partnership NHS Foundation Trust

${ }^{*}$ Corresponding author.

doi: 10.1192/bjo.2021.506

Aims. The COVID-19 pandemic has presented a challenge in ensuring that routine monitoring can still be offered and occur in a safe and effective manner. Our aim was to continue the regular physical health monitoring of North East Hampshire CMHRS patients who are prescribed lithium during the COVID-19 pandemic, and to achieve above 90\% compliance for the monitoring standards. Lithium monitoring clinics have been established in our CMHRS since 2017, Lithium is a high-risk medication as recognised by a National Patient Safety Alert in 2009. Previous annual POMH-UK audits have identified suboptimal monitoring of Lithium patients at local and national levels.

Method. The 'Plan-Do-Study-Act' (PDSA) approach was utilised and a 'QI Bundle' formed. A database of patients who are prescribed lithium has been created and maintained. Synchronised standardised reminder letters are sent to the patients twice yearly. NICE recommends that patients on lithium have their lithium levels checked every 3 months for the first year and then at least every 6 months, plus TFTs, U\&Es, calcium and weight every 6 months (or more frequently if impaired). The Specialist Pharmacist Service advise that during the COVID-19 pandemic, if patients are not in the at-risk category then monitoring intervals can be extended by up to 3 months, but that patients in the at-risk category should have their normal monitoring intervals continued.

Lithium clinics have been held every April and October since 2017 by the Junior Doctors allocated to NE Hampshire CMHRS. This year they were conducted via telephone appointment or face to face where safe to do so with clinician in full PPE. An audit was subsequently carried out in December 2020 to assess our patient's compliance with the aforementioned NICE recommendations for lithium monitoring, the results of which were compared to previous annual audits.

Result. Seventeen patients were currently prescribed lithium within the NE Hampshire CMHRS. Over 90\% compliance with monitoring was achieved in December 2020 apart from the checking of calcium levels which was slightly below target at $82 \%$, and weight which was at $88 \%$. There was no significant reduction in monitoring standards obtained compared to data from the previous three years.

Conclusion. Routine monitoring for our patients who are prescribed Lithium was effectively and safely continued during the pandemic. Above 90\% compliance with lithium monitoring standards was nearly obtained across all areas. We will continue to offer lithium monitoring twice yearly.

Improving training and support by improving our out of hours handover, Central and North West London NHS Foundation Trust

\author{
Mehmet $\mathrm{Gez}^{*}$ and Guang Xu \\ Central and North West London NHS Foundation Trust \\ ${ }^{\star}$ Corresponding author.
}

doi: $10.1192 /$ bjo.2021.507

Aims. During out of hour handovers at St Charles Hospital - the two duty SHO (senior house officers) cover on site, whereas the on-call registrar and consultant are available to contact by phone. Some trainees may experience difficulties in contacting their seniors for support, or may not feel comfortable doing so. Trainees may also feel like they would benefit from being more informed of the hospital situation, or added learning and educational opportunities from the shift. The aim of this project was to improve the out of hours support for the on-call SHOs - which we hope to have positive short (such as improving confidence and performance) - and longer-term impacts (improving retention in the deanery and specialty). 